\title{
A dysbiotic mycobiome dominated by Candida albicans is identified within oral squamous-cell carcinomas
}

Manosha Perera, Nezar Noor Al-hebshi, Irosha Perera, Deepak Ipe, Glen C. Ulett, David J. Speicher, Tsute Chen \& Newell W. Johnson

To cite this article: Manosha Perera, Nezar Noor Al-hebshi, Irosha Perera, Deepak Ipe, Glen C. Ulett, David J. Speicher, Tsute Chen \& Newell W. Johnson (2017) A dysbiotic mycobiome dominated by Candida albicans is identified within oral squamous-cell carcinomas, Journal of Oral Microbiology, 9:1, 1385369, DOI: 10.1080/20002297.2017.1385369

To link to this article: https://doi.org/10.1080/20002297.2017.1385369
(2) 2017 The Author(s). Published by Informa UK Limited, trading as Taylor \& Francis Group.
Published online: 27 Oct 2017.

Џll Article views: 543

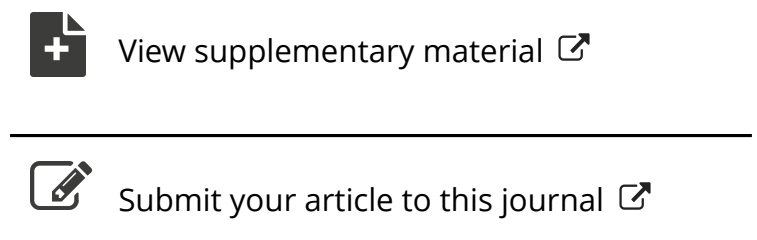

Submit your article to this journal $\sqsubset$

View Crossmark data ¿ 


\title{
A dysbiotic mycobiome dominated by Candida albicans is identified within oral squamous-cell carcinomas
}

\author{
Manosha Pereraa*, Nezar Noor Al-hebshib*, Irosha Pererac, Deepak Ipe ${ }^{\mathrm{d}, \mathrm{e}}$, Glen C. Ulett (D) ${ }^{\mathrm{d}, \mathrm{e}}$, \\ David J. Speicher (iD) ${ }^{\mathrm{e}, \mathrm{f}, \mathrm{g}}$, Tsute Chen ${ }^{\mathrm{h}}$ and Newell W. Johnson ${ }^{\mathrm{a}, \mathrm{e}, \mathrm{i}}$ \\ aSchool of Dentistry and Oral Health, Griffith University, Queensland, Australia; ${ }^{b}$ Maurice H. Kornberg School of Dentistry, Temple \\ University, Philadelphia, USA; 'Preventive Oral Health Unit, The National Dental Hospital (Teaching), Colombo, Sri Lanka; ${ }^{d}$ School of \\ Medical Science, Griffith University, Queensland, Australia; 'Menzies Health Institute Queensland; Griffith University, Nathan, Australia; \\ ${ }^{f}$ Microbiology and Infectious Diseases, St. Joseph's Healthcare Hamilton, Hamilton, Canada; ${ }^{9}$ Department of Pathology and Molecular

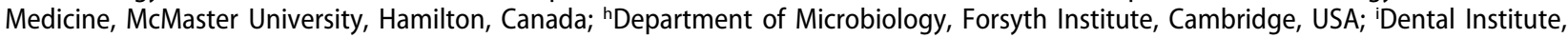 \\ King's College London, London, United Kingdom
}

ABSTRACT

The aim of this study was to characterize the mycobiome associated with oral squamous-cell carcinoma (OSCC). DNA was extracted from 52 tissue biopsies (cases: 25 OSCC; controls: 27 intra-oral fibro-epithelial polyps [FEP]) and sequenced for the fungal internal transcribed spacer 2 region using Illumina ${ }^{\mathrm{TM}} 2 \times 300 \mathrm{bp}$ chemistry. Merged reads were classified to species level using a BLASTN-algorithm with UNITE's named species sequences as reference. Downstream analyses were performed using QIIME $^{\mathrm{TM}}$ and linear discriminant analysis effect size. A total of 364 species representing 160 genera and two phyla (Ascomycota and Basidiomycota) were identified, with Candida and Malassezia making up $48 \%$ and $11 \%$ of the average mycobiome, respectively. However, only five species and four genera were detected in $\geq 50 \%$ of the samples. The species richness and diversity were significantly lower in OSCC. Genera Candida, Hannaella, and Gibberella were overrepresented in OSCC; Alternaria and Trametes were more abundant in FEP. Species-wise, Candida albicans, Candida etchellsii, and a Hannaella luteola-like species were enriched in OSCC, while a Hanseniaspora uvarum-like species, Malassezia restricta, and Aspergillus tamarii were the most significantly abundant in FEP. In conclusion, a dysbiotic mycobiome dominated by $C$. albicans was found in association with OSCC, a finding worth further investigation.
ARTICLE HISTORY

Received 8 April 2017

Accepted 7 September 2017

\section{KEYWORDS}

Fungi; mycobiome;

carcinoma; high-throughput nucleotide sequencing; DNA

ribosomal spacer; microbiome; mouth; squamous cell

\section{Introduction}

Oral squamous-cell carcinoma (OSSC) accounts for $>90 \%$ of cases of oral cancer. OSSC is a malignancy with poor prognosis, representing a public health challenge, particularly in less developed regions where it ranks as the 8 th most common cancer type [1-3]. The incidence rates are exceptionally high in certain countries such as Papua New Guinea, India, Sri Lanka, the Maldives, and Pakistan [1]. There are a number of wellestablished risk factors for OSSC, including smoking, smokeless tobacco usage, areca nut and betel quid chewing, alcohol consumption, and human papillomavirus infections $[4,5]$. However, around $15 \%$ of oral cancer cases are not attributable to any of these. The role of other factors such as infection and inflammation is thus emerging [6,7].

The oral cavity harbors the second most diverse microbiota in our body: the oral microbiome [8]. Oral microorganisms, predominantly bacteria, form homeostatic communities that live in a mutualistic relationship with the host. However, local ecological challenges may disturb the community balance and result in a microbial 'dysbiosis' characterized by altered microbiome profile and the potential to cause or contribute to disease [9]. Technological advances, particularly the advent of nextgeneration sequencing (NGS), have revolutionized the study of the oral microbiome in health and disease [10]. Periodontitis and dental caries, the most common oral diseases, are recognized today as sequelae of oral microbial dysbiosis $[9,11]$. Recent evidence from NGS studies also suggest a possible role of a dysbiotic microbial community in OSCC $[7,12]$. However, all these studies have focused only on the bacterial component of the microbiome (i.e. the bacteriome).

Numerous fungi are found in the oral cavity, with the genus Candida being the most commonly isolated and thus studied. Recent studies using NGS have revealed the presence of a complex fungal community (the mycobiome) in healthy individuals. In 2010, a study by Ghannoum et al. revealed the presence of a core oral mycobiome consisting of 13 taxa, with Candida, Saccharomycetales, Cladosporium, and 
Aspergillus being the most abundant [13]. In 2014, Dupuy et al. [14] identified an additional five core genera, of which Malassezia was detected for the first time in the oral cavity and was even found to be more abundant than Candida in some of the subjects. So far, no attempts have been made to explore the potential role of the oral mycobiome in oral health and disease, including oral cancer. This is surprising, given the existing evidence implicating Candida albicans in oral carcinogenesis [15].

The objective of this study was to use NGS coupled with a species-level taxonomy assignment algorithm to compare the mycobiome profile within OSSC tissues to benign intra-oral fibro-epithelial polyps (FEP) and to identify fungal taxa that may play a role in oral carcinogenesis.

\section{Methods}

\section{Study design, setting, and subjects}

This was a case-control study. Subjects were recruited between 17 April 2015 and 2 August 2015 at nine oral and maxillofacial (OMF) units in six provinces of Sri Lanka. Cases comprised 25 Sinhala, $\geq 40$-year-old males with histologically confirmed OSCC affecting the buccal mucosa or tongue. The control group consisted of 27 Sinhala males with a clinical diagnosis of FEP also involving the buccal mucosa or tongue. Subjects with a history of antibiotic use in the last 2 months were excluded.

Ethical approval for the study was obtained from the Faculty Research Committee, Faculty of Dental Sciences, University of Peradeniya, Sri Lanka (FRC/ FDS/UOP/E/2014/32) and Griffith University Human Research Ethics Committee, Australia (DOH/18/14/ HREC). Written informed consent was obtained from each participant.

\section{History taking and clinical examination}

Data were collected using a pretested, intervieweradministered questionnaire, which comprised information on socio-demographics and risk habits, including use of smokeless tobacco, areca nut and betel quid chewing, tobacco smoking, and alcohol consumption. Clinical oral examinations were conducted by dental public-health specialists. The oral mucosa was thoroughly inspected for any growth, ulceration, or white patches. The number of missing teeth was recorded. Oral hygiene status was assessed with the simplified oral hygiene index [16], while periodontal status was assessed using bleeding on probing (BOP), periodontal pocket depth (PPD), and clinical attachment loss (CAL) at four sites per anterior tooth and six sites per posterior tooth.

\section{Tissue sampling and DNA extraction}

For suspected OSSC cases, tissue samples were obtained from the incisional biopsies taken for diagnosis. The freshly taken biopsy was laid on a pile of sterile gauze, and a small piece of tissue $\left(\sim 3 \mathrm{~mm}^{3}\right)$ was excised from the deep tissue at the macroscopically visible advancing front of the neoplasm, avoiding contamination from the tumor surface. A new sterile surgical blade was used for each case. The sample was aseptically transferred into a screw-cap vial and placed in a polystyrene box containing dry ice. These were transferred to a $-80^{\circ} \mathrm{C}$ freezer in a university laboratory as soon as practicable. Simultaneously, the rest of the biopsy was sent in $10 \%$ buffered formalin for histopathological diagnosis. Only samples histopathologically confirmed as OSSC were included in the study. Control tissue samples were obtained as above from freshly excised, clinically diagnosed FEPs.

Tissue samples ( 100 mg each) were finely chopped using a sterile blade. DNA extraction was then performed using Gentra Puregene Tissue kit (Qiagen, Hilden, Germany), according to the manufacturer's instructions (solid tissue protocol) with a few modifications: (1) incubation in the lysis buffer was performed overnight; and (2) an additional lysis step using 50 units of mutanolysin at $37^{\circ} \mathrm{C}$ for $1.5 \mathrm{~h}$ to digest the cell wall of Gram + bacteria was included prior to the addition of Proteinase K (the samples had also been planned to be analyzed for bacterial content). Total DNA concentration and purity were determined using the NanoDrop ${ }^{\mathrm{Tw}}$ 1000 Spectrophotometer (Thermo Fisher Scientific, Waltham, MA). The extracts were stored at $-80^{\circ} \mathrm{C}$.

\section{Fungal load}

The fungal load was assessed by quantification of the ribosomal internal transcribed spacer 2 (ITS2) normalized to the human $\beta$-actin gene using SYBR Greenbased real-time polymerase chain reaction (PCR) and the $2^{-\Delta \Delta \mathrm{Ct}}$ method. The primers ITS3-F (5'-GCATCGATGAAGAACGCAGC-3') and ITS4-R (5'-TCCTCCGCTTATTRATATGC-3') [17] were used to target ITS2, while primers $\beta$-actin-gDNA-F (5'TCCGCAAAGACCTGTACGC- $3^{\prime}$ ) and $\beta$-actingDNA-R (5'-CAGTGAGGACCCTGGATGTG-3') were used to amplify the $\beta$-actin gene. Each reaction comprised $5 \mu \mathrm{L}$ SYBR Green mastermix, $0.2 \mu \mathrm{L}$ primer mix $(10 \mu \mathrm{M}), 2.8 \mu \mathrm{L}$ water, and $2 \mu \mathrm{L}$ template DNA diluted 1:320. Amplification was carried on a ViiA7 platform (Applied Biosystems, Foster City, CA), including an initial enzyme activation cycle at $95^{\circ} \mathrm{C}$ for of $10 \mathrm{~min}$ followed by 50 cycles of denaturation at $95^{\circ} \mathrm{C}$ for $15 \mathrm{~s}$, annealing at $55^{\circ} \mathrm{C}$ for $30 \mathrm{~s}$, and extension at $60^{\circ}$ $\mathrm{C}$ for $60 \mathrm{~s}$. 


\section{Amplicon library preparation and sequencing}

An amplicon library was prepared as per the Illumina's protocol, except that amplification was performed with the Q5 Hot Start High-Fidelity 2× MasterMix (New England Biolabs, Ipswich, MA). In brief, ITS2 was amplified using the primers ITS3-F and ITS4-R, linked to Illumina's specific adapter sequences in standard PCR conditions. The resultant PCR amplicons ( 250-590 bp) were purified using Agencourt AMPure XP beads (Beckman Coulter, Brea, CA). A second PCR was performed to tag the amplicons with unique eight-base barcodes using Nextera XT v2 Index Kit sets A-D (Illumina, San Diego, CA). A set of negative amplification controls (mastermix alone and with other reaction components) were included for both the amplicon production and indexing reactions. The tagged amplicons were then pooled together in equimolar concentrations and sequenced on a MiSeq Sequencing System (Illumina) using v3 $2 \times 300 \mathrm{bp}$, paired-end sequencing chemistry in the Australian Centre for Ecogenomics, according to the manufacturer's protocol.

\section{Processing of sequencing data}

Raw sequencing data were deposited in (and are publicly available from) Sequence Reads Archive (SRA) under project no. PRJNA375780. Reads with primer mismatches were removed, and primer sequences were trimmed off. Paired sequences were then merged with PEAR [18] using the following parameters: minimum amplicon length, $213 \mathrm{bp}$; maximum amplicon length, $552 \mathrm{bp}$; and $p$-value, 0.001. Preprocessing of the merged reads was performed using mothur v1.38.1 [19]. First, to minimize sequencing errors stringently, reads with ambiguous bases, reads with homopolymers $>8$ bp, or reads that did not achieve a sliding 50nucleotide Q-score average of $\geq 30$ were filtered out. Second, the high-quality reads were cleared of chimeras with Uchime [20] using the self-reference approach [21]. Finally, sequences representing nonfungal lineages, identified by preliminary taxonomy using mothur's classify.seqs command, were removed.

\section{Taxonomy assignment algorithm and downstream analysis}

The high-quality, non-chimeric merged reads were classified at the species level employing a previously described BLASTN-based algorithm, modified to analyze the fungal ITS2 region instead of the bacterial 16S rRNA gene [22]. A set of 23,423 fungal ITS sequences representing all named species (16,595 species) in UNITE's database v7.1 (https://unite.ut.ee/repository. php; 22 August 2016 dynamic release; untrimmed sequences) [23] was used as reference (the fasta and taxonomy files of this set can be downloaded at ftp:// www.homd.org/publication_data/20170221/). Briefly, the reads were individually BLASTN searched against the reference set at an alignment coverage of $\geq 99 \%$ and a percent identity of $\geq 98.5 \%$. Hits were ranked by percent identity and, when equal, by bit score. Reads were assigned taxonomies of the best hits. Reads with the best hits representing more than one species were screened again for chimeras using a de novo check at 98\% similarity with USEARCH v8.1.1861 and, if not chimeric, were assigned multiple-species taxonomy [24]. Reads with no matches at the specified criteria underwent secondary de novo chimera checking as above, and then de novo, species-level operational taxonomy unit (OTU) calling at 98\% using USEARCH. Singleton OTUs were excluded; the rest were considered potentially novel species, and a representative read from each was BLASTN-searched against the same reference sequence set again to determine the closest species for taxonomy assignment.

Downstream analysis was performed, as previously described [22]. In short, Quantitative Insights Into Microbial Ecology $\left(\right.$ QIIME $\left.^{\mathrm{mM}}\right)$ v1.9.1 [25] was employed to perform further analysis, including generation of taxonomy plots, rarefaction, calculation of species richness and diversity indexes, computing distance matrixes, and running principle component analysis (PCoA). Detection of differentially abundant taxa between the cases and controls was done using linear discriminant analysis effect size (LEfSe) [26].

\section{Results}

Four samples ended up with a low read count $(<3,000)$ and one with very high count (an outlier) and were thus excluded. Results are presented hereafter for 22 cases and 25 controls. The clinical characteristics of both groups are presented in Table 1. The cases, albeit older on average than controls, were heavier users of betel quid and alcohol, and smoked more, these being the traditional risk factors for oral cancer. They had significantly poorer oral hygiene, fewer teeth, and more severe periodontal disease.

\section{DNA extracts, fungal load, and sequencing/data processing statistics}

Total DNA extracted averaged $482.08 \mathrm{ng} / \mu \mathrm{L}$ (range $16.85-1,908 \mathrm{ng} / \mu \mathrm{L} ;<100 \mathrm{ng} / \mu \mathrm{L}$ from $8 / 47$ [17\%] samples), with all samples containing DNA of high purity (average 260/280 ratio was 1.93; range 1.81-2.07). However, the mean 260/230 ratio was 1.54 (range $0.41-2.22 ; 260$ ) indicating the presence of inhibitors in some of the samples, an issue that was dealt with using dilution before amplification. The average fungal load was 2.4 ITS copies per 1,000 $\beta$-actin gene 
Table 1. Clinical characteristics of the study subjects.

\begin{tabular}{|c|c|c|}
\hline Variable & Cases $(n=22)$ & Controls $(n=25)$ \\
\hline Age $(M \pm S D)^{*}$ & $61.00 \pm 9.5$ & $50.58 \pm 13.5$ \\
\hline$\%$ males & 100 & 100 \\
\hline \multicolumn{3}{|l|}{ Betel quid: $n(\%)^{* *}$} \\
\hline Never & $0(0.0)$ & $4(16)$ \\
\hline Past & $3(13.6)$ & $2(08)$ \\
\hline Sometimes & $1(4.6)$ & $7(28)$ \\
\hline Daily & $18(81.9)$ & $12(48)$ \\
\hline \multicolumn{3}{|l|}{ Smoking: $n(\%)^{* *}$} \\
\hline Never & $4(18.2)$ & $8(32)$ \\
\hline Past & $5(22.7)$ & $7(28)$ \\
\hline Sometimes & $4(18.2)$ & $4(16)$ \\
\hline Daily & $9(40.9)$ & $6(24)$ \\
\hline \multicolumn{3}{|l|}{ Alcohol use: $n(\%)^{* *}$} \\
\hline Never & $0(0.0)$ & $4(16)$ \\
\hline Past & $5(22.7)$ & $2(8)$ \\
\hline Sometimes & $4(18.2)$ & $15(60)$ \\
\hline Daily & $13(59.1)$ & $4(16)$ \\
\hline \multicolumn{3}{|l|}{ Oral hygiene: $n(\%)^{* *}$} \\
\hline Bad & $6(27.3)$ & $3(12)$ \\
\hline Fair & $14(63.6)$ & $10(40)$ \\
\hline Good & $2(9.1)$ & $12(48)$ \\
\hline Missing teeth $(M \pm S D)^{*}$ & $14.73 \pm 10.01$ & $3.85 \pm 4.82$ \\
\hline \multicolumn{3}{|l|}{ Periodontal status: $n(\%)^{* *}$, } \\
\hline Severe periodontitis & 8 (36.4) & $2(08)$ \\
\hline Moderate periodontitis & $11(50.0)$ & $6(24)$ \\
\hline No or mild periodontitis & $3(13.6)$ & $17(68)$ \\
\hline \multicolumn{3}{|l|}{ Site affected: $n(\%)$} \\
\hline Tongue & $14(63.6)$ & $19(76)$ \\
\hline Buccal mucosa & 8 (36.4) & $6(24)$ \\
\hline \multicolumn{3}{|l|}{ Histopathology: $n$ (\%) } \\
\hline Well-differentiated SCC & $11(50)$ & - \\
\hline Moderately differentiated SCC & $11(50)$ & - \\
\hline Fibro-epithelial polyp & - & $25(100)$ \\
\hline
\end{tabular}

${ }^{a}$ Classification made according to Page and Eke [27].

${ }^{*} p \leq 0.05$ ( $t$-test);

${ }^{* *} p \leq 0.05$ (chi-square test).

SSC, squamous-cell carcinoma.

copies (range 0.06-135). This did not significantly differ between the cases and controls.

A few sequences were detected in the negative control, indicating very low background noise (see Supplementary DataSet 1). Sequencing generated $1,576,427$ raw paired reads: $13.6 \%$ of these were discarded due to primer mismatches, and $97.4 \%$ of the remaining reads were successfully stitched with PEAR. Quality filtration and chimera checking removed $7.9 \%$ of the merged reads, thus leaving $1,063,430$ reads $(67.5 \%), 205-535$ bp long. Of these, $1,017,131$ reads $(95.7 \%)$ were successfully classified to the species level; $2.3 \%$ did not return BLASTN matches, and $2 \%$ formed singleton OTUs and were excluded. The number of classified reads per sample averaged 21,641 (range 3,973-54,849).

\section{Overall mycobiome profile}

A total of 364 species belonging to 162 genera and two phyla were detected in the samples. The relative abundances and detection frequencies of these taxa in each of the samples and across the study groups are presented in Supplementary Data Sets 2-4. The number of species per sample ranged from 4 to 64 . However, only 74 genera and 125 species were identified in more than one sample: seven genera and 10 species in $\geq 25 \%$ of the samples, and four genera and five species in $>50 \%$.

The relative abundances of the two phyla identified, as well as the genera and species detected in $\geq 15 \%$ of the samples, are shown in Figure 1. On average, the phyla Ascomycota and Basidiomycota accounted for $78.4 \%$ and $21.6 \%$ of the mycobiome, respectively. At the genus level, Candida was detected in $100 \%$ of the samples and constituted $48 \%$ of the average mycobiome. The genera Malassezia, Cladosporium, and Aspergillus were identified in $\geq 75 \%$ of the samples, with an average relative abundance of $11 \%, 6.1 \%$, and $3.7 \%$, respectively. With regard to species, C. albicans was found in all samples at a mean relative abundance of $44.4 \%$. Malassezia restricta, Aspergillus penicillioides, and Malassezia globosa were identified in $83 \%, 70.2 \%$, and $68.1 \%$ of the samples, respectively, and accounted for $3.2 \%, 2.2 \%$, and $4.2 \%$ of the average mycobiome, respectively. Cladosporium exasperatum and a potentially novel species close to Cladosporium sphaerospermum were also identified in half of the samples at an average relative abundance of $>2 \%$.

However, the relative abundance of these taxa varied significantly between the samples. In addition, the mycobiome of some of the samples was dominated by taxa other than those mentioned above. Examples of species abundant in single samples include Rhodotorula mucilaginosa, Sporidiobolus johnsonii, Penicillium toxicarium, Toxicocladosporium irritans, Gibberella intricans, Alternaria infectoria, Ophiocordyceps sinensis, and Aspergillus tamarii, as well as a number of potentially novel taxa.

\section{OSCC versus FEP}

The number of species per sample ranged from 4 to 29 for the cases and from 8 to 64 for the controls. The FEP controls had significantly higher species richness and $\alpha$-diversity than the cases (Table 2). Rarefaction curves show that as few as 1,500 reads per sample represented sufficient sequencing depth (Figure 2a). No separate clusters formed for the cases and controls by PCoA (Figure 2b).

The genera and species found by LEfSe to be differentially abundant between the cases and controls are shown in Figure 3. The genera Candida, Hannaella, and Gibberella were significantly more abundant in OSCC. In contrast, Trametes and Alternaria were strongly associated with FEP. At the species level, $C$. albicans, C. etchellii, and a potentially novel species close to Hannaella luteola were significantly enriched in OSCC. C. albicans was identified in $100 \%$ of the samples, but the average relative abundance in OSCC was twice that in the controls $(61.2 \%$ vs. $29.6 \%)$. C. etchellsii was identified in $32 \%$ of the cases at a relative abundance of $0.006-2.56 \%$ compared to only $8 \%$ of 


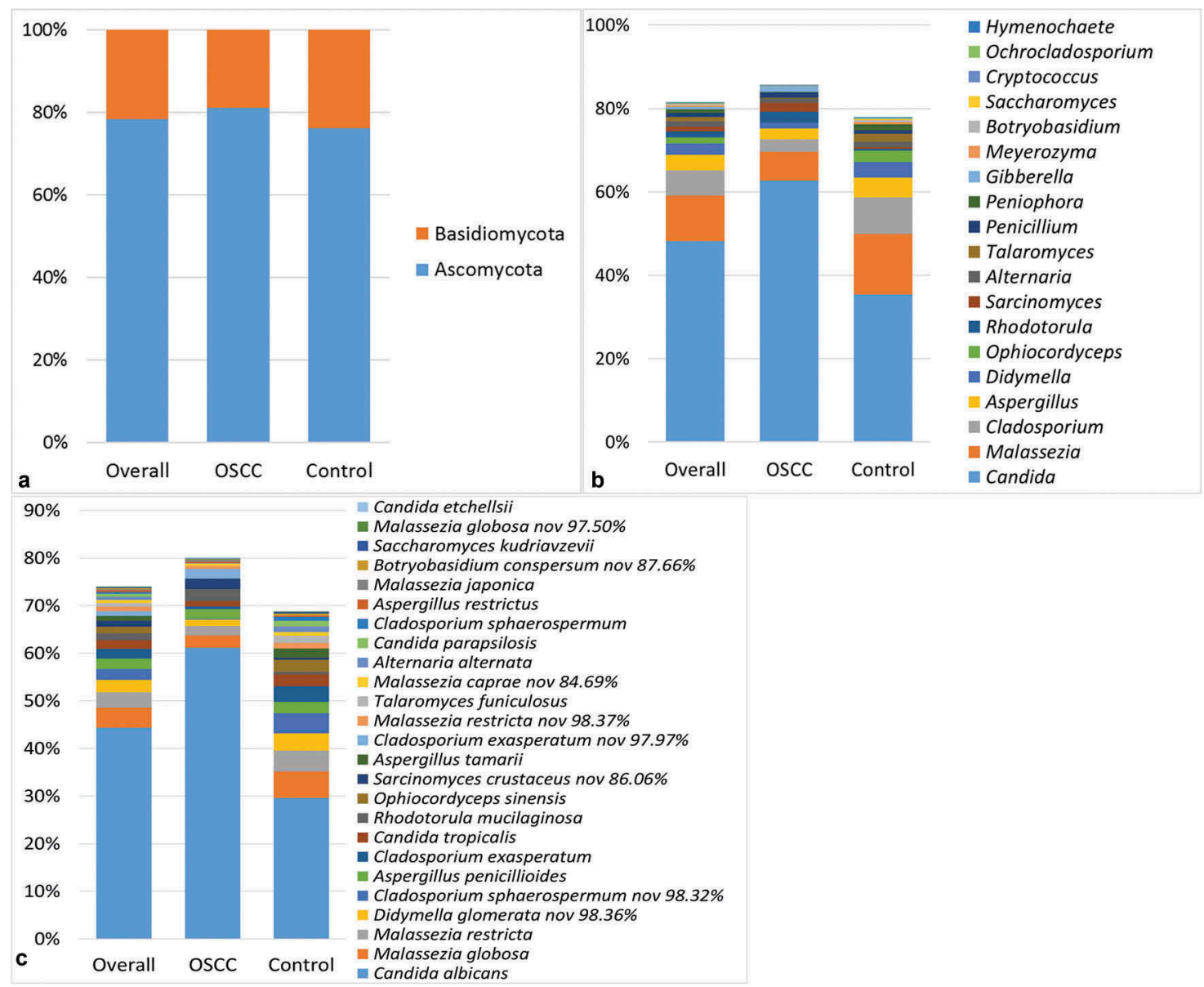

Figure 1. Mycobiome profile. Average relative abundances of the two phyla identified (a) as well as the genera (b) and species (c) detected in $\geq 15 \%$ of the samples.

Table 2. Species richness, a-diversity, and coverage $(M \pm S E)$ calculated from the rarefied biom.

\begin{tabular}{lcccc}
\hline Group & $\begin{array}{c}\text { Observed } \\
\text { richness* }\end{array}$ & \multicolumn{1}{c}{ Chao1* $^{*}$} & $\begin{array}{c}\text { Shannon } \\
\text { index* }\end{array}$ & $\begin{array}{c}\text { Good's } \\
\text { coverage }\end{array}$ \\
\hline OSCC & $11.7 \pm 6.4$ & $13.6 \pm 7.0$ & $1.5 \pm 0.9$ & $0.999 \pm 0.000$ \\
FEP & $17.7 \pm 9.8$ & $19.9 \pm 12.2$ & $2.1 \pm 1.2$ & $0.999 \pm 0.001$ \\
\hline
\end{tabular}

${ }^{*} p \leq 0.05$ (Mann-Whitney test).

OSCC, oral squamous-cell carcinoma; FEP, fibro-epithelial polyps. the controls at a relative abundance of $0.007-0.12 \%$. The H. luteola-like species was detected in $20 \%$ of OSCC samples versus none of the controls. On the other hand, a potentially novel Hanseniaspora uvarum-like species, in addition to M. restricta, A. tamarii, Cladosporium halotolerans, Alternaria alternata, and Malassezia furfur were overrepresented or even exclusively found in FEP (see Supplementary Table S1 for a list of taxa exclusively found in either group at a prevalence of $\geq 10 \%$ ).
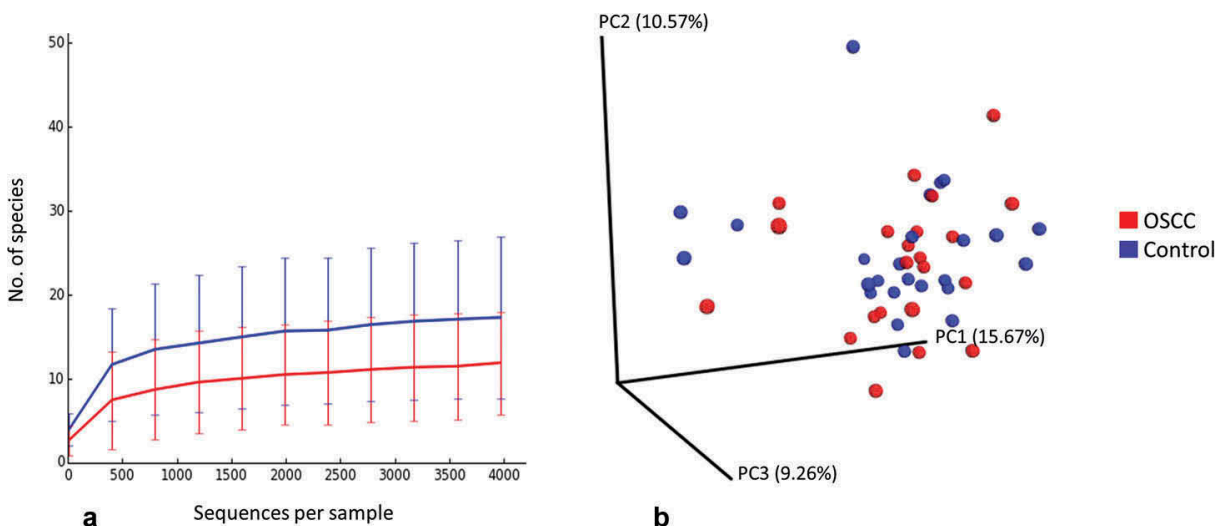

Figure 2. Rarefaction and $\beta$-diversity. (a) Rarefaction curves showing the number of observed species as a function of sequencing depth. (b) Non-clustering of the study subjects by principal components analysis (weighted Unifrac). 

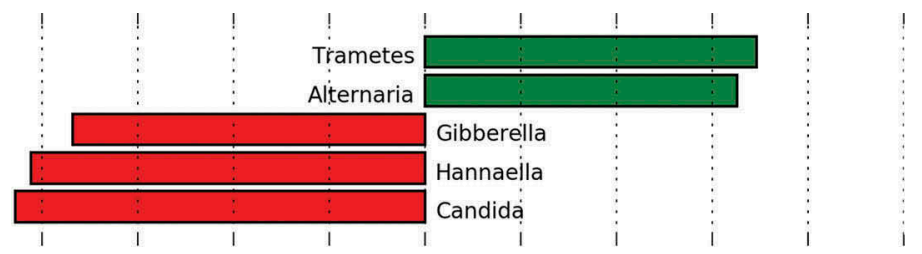

a

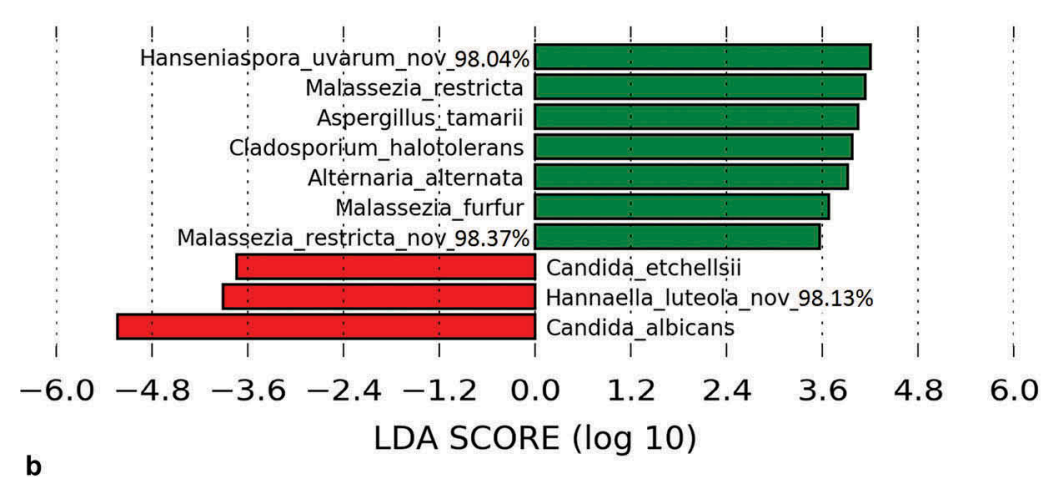

OSCC

Control

Figure 3. Differentially abundant taxa. Linear discriminant analysis effect size (LEfSe) analysis showing genera (a) and species (b) that were significantly differentially abundant between the cases and controls (LDA score $\geq 3$ ). The differences were also found to be significant by the Mann-Whitney test.

\section{Discussion}

To the authors' knowledge, this is the first study to explore the mycobiome associated with oral cancer. The study subjects were recruited from Sri Lanka, a developing lower-middle income country where oral cancer is the most common malignancy among men [1]. Deep-tissue biopsies, rather than surface swabs or saliva, were used to ensure that any association identified was more relevant to carcinogenesis. Unlike previous studies, OSCC was compared with FEP instead of buccal epithelium or adjacent normal tissue (i.e. malignant vs. benign growths). This is a unique approach that is arguably a strength of the study. However, since FEP is diagnosed at a younger age, a significant difference in age was inevitable. OSCC patients were also heavier users of tobacco and of betel nut and alcohol and had more severe periodontitis. These are all established risk factors of OSCC, and it would have been impossible to match the controls for them or to adjust for their confounding effects statistically, given the small sample size. Nevertheless, these factors may have accounted in part for the microbial differences observed-an inherent limitation that has to be acknowledged. There are other limitations to note. First, some DNA samples had low 260/230 ratios, suggestive of the presence of inhibitors. However, these samples amplified successfully after dilution. Second, the DNA extraction protocol was not optimized to maximize recovery of fungal DNA from the tissue samples, which may have resulted in underestimation of the fungal loads. Finally, a few reads were detected in the control samples, which, while having negligible effect on the results, suggested some sort of contamination at some stage of the laboratory work. The latter could have been due to contamination of the DNA extraction or sequencing reagents by extraneous organisms, now known to be a problem around the world [28].

Coupling Illumina's $2 \times 300$ bp sequencing chemistry and merging of paired reads ensured that full ITS2 amplicons were obtained, maximizing taxonomic resolution. This, in addition to limiting the reference set to sequences from named species only, enabled the reads to be classified at the species level. A percent identity threshold of $98.5 \%$ (i.e. like that used to generate species hypothesis in UNITE's dynamic release) and high query alignment coverage $(\geq 99 \%)$ cutoff increased the reliability of taxonomic assignment and eliminated the possibility of forced classifications by BLASTN. However, this approach carries the risk of assigning reads belonging to species not represented in the reference set to another closely related species.

Of the species identified, 239/364 (65.6\%) were from single samples, indicating that they represent transient environmental fungi. Although the scope of the current study is different from that of the previous two oral mycobiome studies in which salivary samples from healthy subjects were analyzed, some comparisons can be made. Ghannoum et al., using a relative abundance cutoff of $1 \%$, identified 101 species in their samples [13]. Applying the same cutoff to our samples left 137 species, which is comparable. They also described a basal mycobiome comprising 13 taxa that were present in $\geq 20 \%$ of the subjects. Consistently, seven of these genera, namely Candida, Aspergillus, Cladosporium, Alternaria, Cryptococcus, Gibberella, and Saccharomyces, were detected at the same frequency in at least one of the two groups. Interestingly, Malassezia, which was not detected by 
Ghannoum et al., was identified in this study as the second most common and abundant genus after Candida. This substantiates the more recent findings by Dupuy et al., who, for the first time, described this genus in the oral cavity and even found it to be more abundant than Candida [14]. The same study described a core mycobiome comprising 14 core genera detected in $\geq 50 \%$ of subjects. Four of these (Candida, Malassezia, Aspergillus, and Cladosporium) were also found in more than half of our samples. Put together, these findings provide evidence for the existence of a resident, core oral fungal community.

Fungi in the oral cavity belong to what is called the rare biosphere [29]. It is therefore not surprising that they were detected in low counts, especially given that deep-tissue samples were analyzed. Regardless, their role in disease should not be discounted, as even in these small number, they can have a substantial effect on the microbiota, functioning as keystone pathogens [30]. There was, however, no significant difference in the fungal load between the cases and controls, which is inconsistent with the study by Berkovits et al. [31], who found the 'fungal burden' to be higher in OSCC patients compared to healthy controls. However, this may be explained, at least in part, by the fact that surface swabs rather than tissue samples were used in that study: more fungi are likely to be present on the rough tumor surface compared to the smooth surface of the healthy mucosa. In addition, the authors in that study used a culturebased approach rather than quantitative PCR, which may have resulted in missing some difficult-to-grow species. Indeed, Malassezia, which is a dominant taxon in many of the samples in this study, was not detected in the study by Berkovits et al. [31]. As discussed below, the differences between OSCC and FEP in this study were in terms of relative abundances.

Candida, specifically C. albicans and C. etchellsii, showed the strongest association with OSCC in the current study. In fact, candidiasis has for long been proposed as a risk factor for malignant transformation of oral potentially malignant disorders, including leukoplakia [32]. Some strains of C. albicans have high nitrosation potential and have been experimentally shown to induce dysplasia [33]. Recently, there has been increasing evidence of the association between candida infection and OSCC [15]. Nagy et al. recovered C. albicans from the surface of $8 / 21$ (38\%) neoplasms but from none of the control samples [34]. Similarly, Čanković et al. isolated Candida from 9/30 (four $C$. albicans and five non-albicans) cancer surfaces (30\%) but from the surface of only 2/30 (6.6\%) benign growths [35]. Gall et al. identified Candida spp. in 31/48 (65\%) oral cancer cases, but the study did not include healthy control samples for comparison [36]. More recently, Berkovits et al. isolated yeasts, predominantly Candida spp., from the surface of 18/20 (90\%) tumors compared to only $12 / 40(30 \%)$ healthy mucosae [31]. In the present study, C. albicans was detected in $100 \%$ of the samples, which is probably a reflection of the much higher sensitivity of NGS compared to cultural techniques used in previous studies. The difference, however, between the cases and controls in the current material was in terms of relative abundance (average of $61.2 \%$ in the cases vs. $29.6 \%$ in the controls).

C. etchellsii was identified in $32 \%$ of the cases vs. $8 \%$ of the controls. This is the first time a specific Candida species other than C. albicans has been implicated in oral cancer, a finding worth further investigation. Hannaella and Gibberella spp. were also overrepresented in OSCC tissues. These are typically found on plants as commensals and pathogens, respectively $[37,38]$. Therefore, they probably represent contaminants of the OSCC rather than members of the oral mycobiome community, especially considering that they were found in only one control sample. However, their contribution to the carcinogenic process cannot be excluded. In fact, one Gibberella spp. (G. moniliformis) produces a mycotoxin that has been shown to induce liver cancer in rodents [39].

Species belonging to Malassezia, Aspergillus, Alternaria, Cladosporium, and Hanseniaspora were significantly enriched or exclusively found in the controls. Malassezia spp. are normal colonizers of healthy skin [40] and have been recently found to be dominant members of the salivary mycobiome [14]. Aspergillus, Alternaria, and Cladosporium have also been described as core oral fungal taxa $[13,14]$. Interestingly, some species of these genera, including $A$. tamarii and A. alternata, which were identified in the current samples, are known to produce compounds with anticancer activity [41-44]. In addition, statins produced by $A$. tamarii have been shown to inhibit growth of $C$. albicans [43], which possibly explains why all samples with high relative abundance of this species had very low levels of C. albicans (Supplementary Data Set 3). Therefore, while these species may simply represent transient environmental fungi or passenger oral fungal taxa, it is also possible that carriage of some of these species confers some protection against the development of oral cancer. Further research to explore these scenarios is needed to harness their potential for novel prevention and control strategies.

In conclusion, the current study uncovered a dysbiotic mycobiome characterized by lower species diversity and increased C. albicans relative abundance in association with OSCC. It also identified a number of fungal taxa capable of producing anticancer compounds exclusively in non-cancerous tissues. The relevance of these findings for development of risk markers and for prevention and control of OSCC warrants further investigation.

\section{Acknowledgments}

We acknowledge Prof. WM Tilakaratne, Senior Professor of Oral Pathology, the Dean, Faculty of Dental Sciences, 
University of Peradeniya, Sri Lanka, and Prof. L. Samaranayake, Honorary Professor of Oral Microbiomics and Infection, School of Dentistry, University of Queensland, for valuable guidance. We thank Oral and Maxillo-Facial Surgeons Dr. Sharika Gunathilake, Dr. S.A.K.J. Kumara, Dr. Ranjith Lal Kandewatte, Dr. P. Kirupakaran, Dr. D.K. Dias, Dr. Chamara Athukorale, Dr. Suresh Shanmuganathan, and Dr. T. Sabesan for facilitating data and sample collection from their respective Oral and Maxillo-Facial Units.

\section{Disclosure statement}

No potential conflict of interest was reported by the authors.

\section{Funding}

This work was supported by the The Australian Research Council; Self-finance (M.P. and I.P.); Griffith University Higher Degree Scholarships for international students.

\section{Notes on contributors}

Manosha Perera is a PhD Candidate of School of Dentistry and Oral Health, Griffith University, Goldcoast. Her current research is on Metagenomics and Microbiomics in Cancer Epidemiology. She has obtained BSc. and MSc. in Applied Microbiology from University of Kelaniya, Sri Lanka. She has 23-year- experience in all aspects of Microbiological Diagnostic Techniques at the Medical Research Institute, Colombo, Sri Lanka.

Nezar Noor Al-hebshi is a Research Associate Professor and Co-director of the Oral Microbiome Research Laboratory at Temple University Kornberg School of Dentistry, and a primary member of Fox Chase Cancer Center. He obtained his $\mathrm{PhD}$ from University of Bergen, Norway. His research currently focuses on two areas: 1) role of the microbiome in oral squamous cell carcinoma as well; and 2) use of in vitro microbiome models to screen for microbiome modulators.

Irosha Perera is a Dental Public Health Specialist of the Preventive Oral Health Unit, National Dental Hospital (Teaching) Sri Lanka. She received a merit award from the Postgraduate Institute of Medicine, University of Colombo, Sri Lanka for her thesis on social inequalities in dental caries among adolescents submitted for the Doctor of Medicine in Community Dentistry Examination held in 2006. Her research interests span from public health perspectives to metagenomics and microbiomics as applied to Head and Neck Cancer.

Deepak S. Ipe is a researcher with expertise in Molecular Biology at Griffith University, completed his doctorate degree on "molecular mechanisms of Group B Streptococcus urinary tract infection and adaptability for growth in human urine" in 2014. Currently Deepak Ipe is working on enhancing osteogenesis through 3D printed bio-materials at School of Dentistry and Oral Health, Griffith University.

Glen C. Ulett is Professor of Microbiology, Griffith University. He received his Ph.D. from James Cook University in 2001 and completed postdoctoral research at St. Jude Children's Research Hospital, University of
Queensland, and University of Alabama at Birmingham. His research focuses on bacterial pathogenesis and mechanisms of host defence.

David J. Speicher is a molecular virologist with expertise in molecular diagnostics and microbiomics. He received his PhD from Griffith University, Australia in 2012, and is currently a postdoctoral fellow at St Joseph's Healthcare Hamilton and McMaster University in Hamilton, Ontario, Canada. His research interests include the detection of viruses in saliva as well as the detection of bacteriophages associated with Clostridium difficile.

Tsute Chen is an Associate Research Investigator and senior bioinformatic expert at Forsyth Institute. He obtained his PhD from University of Massachusetts, Amherst, MA, USA. His research focus in the past 10 years has been on the analysis of high-throughput data for oral microorganisms, such as genomic sequences, microarray, and NGS transcriptomic profiles. His group has developed many useful bioinformatics tools which can be found on public web sites such as the Bioinformatics Resource for Oral Pathogens (BROP) and The Human Oral Microbiome Database (HOMD).

Newell Johnson has over 50 years of teaching, research and contributions to professional organizations covering oral pathology, oral medicine, and periodontology, and the study of dental and medical education. These have been conducted from the Universities of Melbourne, Bristol and London, and the Royal College of Surgeons of England. He was Foundation Dean of the School of Dentistry and Oral Health at Griffith University in Queensland, Australia where, as Professor Emeritus, he currently leads a team of $\mathrm{PhD}$ students and colleagues from many countries in both basic and translational research into the above areas. He has over 400 refereed publications $\mathrm{He}$ is the recipient of distinguished scientist awards from the IADR and from professional associations in Britain and several other countries, as well as Civil Honours for his contributions to international public health.

\section{ORCID}

Glen C. Ulett (D) http://orcid.org/0000-0002-9638-8091

David J. Speicher (D) http://orcid.org/0000-0002-1745-3263

\section{References}

[1] Ferlay J, Soerjomataram I, Ervik M, et al. GLOBOCAN 2012 v1.0, cancer incidence and mortality worldwide: IARC CancerBase No. 11 [Internet]. Lyon: International Agency for Research on Cancer; 2013. [cited 2013]. Available from: http://globocan.iarc.fr

[2] Warnakulasuriya S. Global epidemiology of oral and oropharyngeal cancer. Oral Oncol. 2009;45:309-316. Epub 2008 Sep 23.

[3] Wang B, Zhang S, Yue K, et al. The recurrence and survival of oral squamous cell carcinoma: a report of 275 cases. Chin J Cancer. 2013;32:614-618.

[4] Petti S. Lifestyle risk factors for oral cancer. Oral Oncology. 2009;45:340-350.

[5] Gupta B, Johnson NW, Kumar N. Global epidemiology of head and neck cancers: a continuing challenge. Oncology. 2016;91:13-23. 
[6] Chocolatewala N, Chaturvedi P, Desale R. The role of bacteria in oral cancer. Indian J Med Paediatr Oncol. 2011;31:126-131.

[7] Perera M, Al-Hebshi NN, Speicher DJ, et al. Emerging role of bacteria in oral carcinogenesis: a review with special reference to perio-pathogenic bacteria. J Oral Microbiol. 2016;8:32762.

[8] Human Microbiome Project C. Structure, function and diversity of the healthy human microbiome. Nature. 2012;486:207-214.

[9] Kilian M, Chapple IL, Hannig M, et al. The oral microbiome - an update for oral healthcare professionals. Br Dent J. 2016;221:657-666.

[10] Chen H, Jiang W. Application of high-throughput sequencing in understanding human oral microbiome related with health and disease. Front Microbiol. 2014;5:508.

[11] Wade WG. The oral microbiome in health and disease. Pharmacol Res. 2013;69:137-143.

[12] Al-Hebshi NN. Inflammatory bacteriome featuring Fusobacterium nucleatum and Pseudomonas aeruginosa identified in association with oral squamous cell carcinoma. Sci Rep. 2017;7:1834.

[13] Ghannoum MA, Jurevic RJ, Mukherjee PK, et al. Characterization of the oral fungal microbiome (mycobiome) in healthy individuals. PLoS Pathog. 2010;6:e1000713.

[14] Dupuy AK, David MS, Li L, et al. Redefining the human oral mycobiome with improved practices in amplicon-based taxonomy: discovery of Malassezia as a prominent commensal. PLoS One. 2014;9: e90899.

[15] Mohd Bakri M, Mohd Hussaini H, Rachel Holmes A, et al. Revisiting the association between candidal infection and carcinoma, particularly oral squamous cell carcinoma. J Oral Microbiol. 2010; 2:5780.

[16] Greene JC, Vermillion JR. The simplified oral hygiene index. J Am Dent Assoc. 1964;68:7-13.

[17] White JR, Nagarajan N, Pop M. Statistical methods for detecting differentially abundant features in clinical metagenomic samples. PLoS Comput Biol. 2009;5: e1000352.

[18] Zhang J, Kobert K, Flouri T, et al. PEAR: a fast and accurate Illumina Paired-End reAd mergeR. Bioinformatics. 2014;30:614-620.

[19] Schloss PD, Westcott SL, Ryabin T, et al. Introducing mothur: open-source, platform-independent, community-supported software for describing and comparing microbial communities. Appl Environ Microbiol. 2009;75:7537-7541.

[20] Edgar RC, Haas BJ, Clemente JC, et al. UCHIME improves sensitivity and speed of chimera detection. Bioinformatics. 2011;27:2194-2200.

[21] Schloss PD, Gevers D, Westcott SL. Reducing the effects of PCR amplification and sequencing artifacts on 16S rRNA-based studies. PLoS One. 2011;6:e27310.

[22] Al-Hebshi NN. Composition and imputed functions of the bacteriome in smokeless tobacco products with different oral carcinogenicity. Genes. 2017;8:106.

[23] Koljalg U, Nilsson RH, Abarenkov K, et al. Towards a unified paradigm for sequence-based identification of fungi. Mol Ecol. 2013;22:5271-5277.

[24] Edgar RC. Search and clustering orders of magnitude faster than BLAST. Bioinformatics. 2010;26:2460-2461.
[25] Caporaso JG, Kuczynski J, Stombaugh J, et al. QIIME allows analysis of high-throughput community sequencing data. Nature Methods. 2010;7:335-336.

[26] Segata N, Izard J, Waldron L, et al. Metagenomic biomarker discovery and explanation. Genome Biol. 2011;12:R60.

[27] Page RC, Eke PI. Case definitions for use in population-based surveillance of periodontitis. J Periodontol. 2007;78:1387-1399.

[28] Glassing A, Dowd SE, Galandiuk S, et al. Inherent bacterial DNA contamination of extraction and sequencing reagents may affect interpretation of microbiota in low bacterial biomass samples. Gut Pathog. 2016;8:24.

[29] Huffnagle GB, Noverr MC. The emerging world of the fungal microbiome. Trends Microbiol. 2013;21:334-341.

[30] Baker JL, Bor B, Agnello M, et al. Ecology of the oral microbiome: beyond bacteria. Trends Microbiol. 2017;25:362-374.

[31] Berkovits C, Tóth A, Szenzenstein J, et al. Analysis of oral yeast microflora in patients with oral squamous cell carcinoma. SpringerPlus. 2016;5:1257.

[32] Sankari SL, Gayathri K, Balachander N, et al. Candida in potentially malignant oral disorders. J Pharm Bioallied Sci. 2015;7:S162.

[33] O'Grady JF, Reade PC. Candida albicans as a promoter of oral mucosal neoplasia. Carcinogenesis. 1992;13:783-786.

[34] Nagy K, Sonkodi I, Szöke I, et al. The microflora associated with human oral carcinomas. Oral Oncology. 1998;34:304-308.

[35] Čanković M, Bokor-Bratić M. Candida albicans infection in patients with oral squamous cell carcinoma. Vojnosanit Pregl. 2010;67:766-770.

[36] Gall F, Colella G, Di Onofrio V, et al. Candida spp. in oral cancer and oral precancerous lesions. New Microbiol. 2013;36:283-288.

[37] Landell MF, Brandao LR, Barbosa AC, et al. Hannaella pagnoccae sp. nov., a tremellaceous yeast species isolated from plants and soil. Int J Syst Evol Microbiol. 2014;64:1970-1977.

[38] Sidhu GS. Gibberella spp., pathogens of many crop species. In: Sidhu GS, editor. Genetics of plant pathogenic fungi. Advances in plant pathology. Vol. 6. London: Academic Press Limited; 1988. p. 159-167.

[39] Howard PC, Eppley RM, Stack ME, et al. Fumonisin b1 carcinogenicity in a two-year feeding study using F344 rats and B6C3F1 mice. Environ Health Perspect. 2001;109(Suppl 2):277-282.

[40] Prohic A, Jovovic Sadikovic T, Krupalija-Fazlic M, et al. Malassezia species in healthy skin and in dermatological conditions. Int J Dermatol. 2016;55:494-504.

[41] Bladt TT, Frisvad JC, Knudsen PB, et al. Anticancer and antifungal compounds from Aspergillus, Penicillium and other filamentous fungi. Molecules. 2013;18:1133811376.

[42] Lou J, Fu L, Peng Y, et al. Metabolites from Alternaria fungi and their bioactivities. Molecules. 2013;18:58915935.

[43] Nidhiya KA, Satya E, Nitya MR, et al. In vitro anticancer potential of statin from Aspergillus tamarii grd119. Asian J Pharm Clin Res. 2015;8:147-153.

[44] Raj KG, Sambantham S, Manikanadan R, et al. Fungal taxol extracted from Cladosporium oxysporum induces apoptosis in T47D human breast cancer cell line. Asian Pac J Cancer Prev. 2014;15:6627-6632. 